\title{
Rapid Utilization of Telehealth in a Comprehensive Cancer Center as a Response to COVID-19: Cross-Sectional Analysis
}

Peter E Lonergan $^{1^{*}}$, MD, FRCS (Urol); Samuel L Washington III ${ }^{*}$, MD, MAS; Linda Branagan ${ }^{2}$, PhD; Nathaniel Gleason $^{3,4}$, MD; Raj S Pruthi ${ }^{1}$, MD, MHA; Peter R Carroll ${ }^{1}$, MD, MPH; Anobel Y Odisho ${ }^{1,2,3}$, MD, MPH

${ }^{1}$ Department of Urology, Helen Diller Family Comprehensive Cancer Center, University of California, San Francisco, San Francisco, CA, United States

${ }^{2}$ Telehealth Resource Center, University of California, San Francisco, San Francisco, CA, United States

${ }^{3}$ Center for Digital Health Innovation, University of California, San Francisco, San Francisco, CA, United States

${ }^{4}$ Division of General Internal Medicine, University of California, San Francisco, San Francisco, CA, United States

*these authors contributed equally

Corresponding Author:

Anobel Y Odisho, MD, MPH

Department of Urology

Helen Diller Family Comprehensive Cancer Center

University of California, San Francisco

550 16th Street

San Francisco, CA, 94143

United States

Phone: 1 (415) 3532200

Email: anobel.odisho@ucsf.edu

\section{Abstract}

Background: The emergence of the coronavirus disease (COVID-19) pandemic in March 2020 created unprecedented challenges in the provision of scheduled ambulatory cancer care. As a result, there has been a renewed focus on video-based telehealth consultations as a means to continue ambulatory care.

Objective: The aim of this study is to analyze the change in video visit volume at the University of California, San Francisco (UCSF) Comprehensive Cancer Center in response to COVID-19 and compare patient demographics and appointment data from January 1, 2020, and in the 11 weeks after the transition to video visits.

Methods: Patient demographics and appointment data (dates, visit types, and departments) were extracted from the electronic health record reporting database. Video visits were performed using a HIPAA (Health Insurance Portability and Accountability Act)-compliant video conferencing platform with a pre-existing workflow.

Results: In 17 departments and divisions at the UCSF Cancer Center, 2284 video visits were performed in the 11 weeks before COVID-19 changes were implemented (mean 208, SD 75 per week) and 12,946 video visits were performed in the 11-week post-COVID-19 period (mean 1177, SD 120 per week). The proportion of video visits increased from 7\%-18\% to 54\%-72\%, between the pre- and post-COVID-19 periods without any disparity based on race/ethnicity, primary language, or payor.

Conclusions: In a remarkably brief period of time, we rapidly scaled the utilization of telehealth in response to COVID-19 and maintained access to complex oncologic care at a time of social distancing.

(J Med Internet Res 2020;22(7):e19322) doi: 10.2196/19322

\section{KEYWORDS}

health informatics; telehealth; video visits; COVID-19; video consultation; pandemic; electronic health record; EHR

\section{Introduction}

The emergence of the coronavirus disease (COVID-19) pandemic in the United States in March 2020 created unprecedented challenges in the provision of scheduled health care and, in particular, ambulatory cancer care. The rapid spread of COVID-19 has renewed focus on telehealth [1], including video consultations [2], as a means of continuing ambulatory care without increasing the risk of potential exposure for patients, clinicians, and staff. 
Telehealth is the provision of health care remotely by means of a variety of telecommunication platforms such as messaging, audio, and video [3]. While the use of telehealth to deliver cancer care is not new [4] and has already been well described [5], delivering it at the current scale as a result of the COVID-19 pandemic is unprecedented.

University of California, San Francisco (UCSF) Health established a telehealth program in 2015 , which offers video visits in all practices. In response to the evolving pandemic, leadership challenged the organization to transition all in-person clinic visits, beginning March 15, 2020, to video visits with exceptions only for specific, urgent cases.

In this study, we analyze the change in video visit volume at the UCSF Comprehensive Cancer Center in response to COVID-19 and compare demographics and appointment data from January 1, 2020, and in the 11 weeks after the transition to video visits.

\section{Methods}

Demographics and appointment data (dates, visit types, and departments) were extracted from the electronic health record reporting database. The pre-COVID-19 period was defined as the 11 weeks from January 1 to March 13, 2020, prior to the transition, and the post-COVID-19 period as the 11 weeks following the transition to video visits on March 16, 2020, up to May 31, 2020. All video visits were performed using a HIPAA (Health Insurance Portability and Accountability Act)-compliant video conferencing platform (Zoom Video Communications Inc) with a pre-existing workflow (Figure 1). The statistical program $\mathrm{R}$ (version 3.5.3, R Foundation for Statistical Computing) was used for analysis [6] and a $P$ value less than .05 was considered significant.

Figure 1. Video visit workflow used at the University of California, San Francisco.

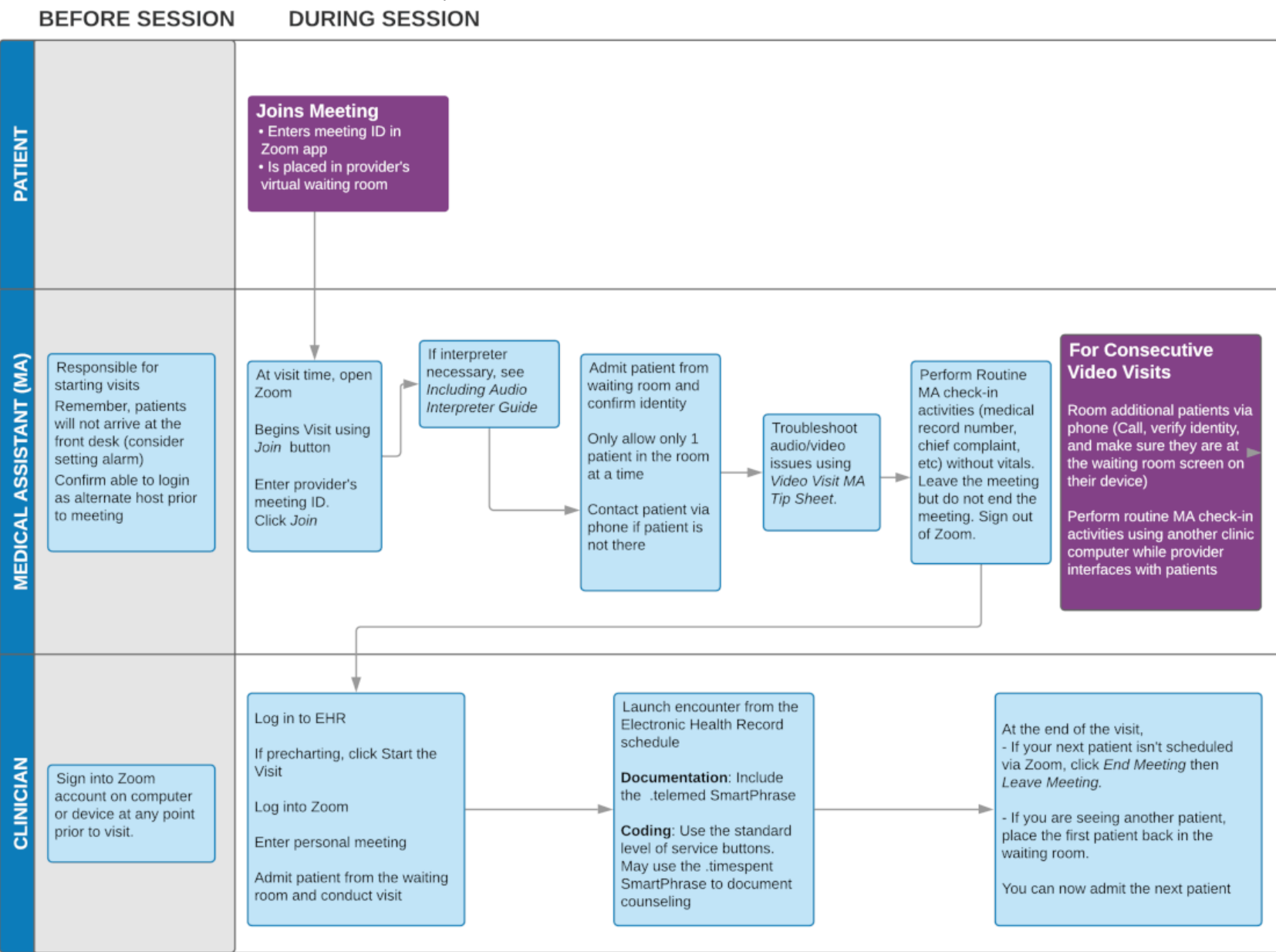

\section{Results}

In the pre-COVID-19 period from January 1 to March 13, 2020, there were a total of 23,988 ambulatory care episodes, with a mean of 2181 (SD 522) episodes per week across 17 departments and divisions at the UCSF Cancer Center (Figure 2). During this period, 2284 video visits were performed, with a mean of 208 (SD 75) video visits being performed per week. The proportion of video visits ranged from $7 \%-18 \%$ whereas the proportion of in-person visits ranged from 76\%-86\% (Figure $3)$. In the post-COVID-19 period from March 16 to May 31, 2020 , there was a total of 20,567 ambulatory care episodes (mean 1870, SD 200 per week). A total of 12,946 video visits were performed in the post-COVID-19 period (mean 1177, SD 120 per week). The proportion of video visits increased to $54 \%-72 \%$. The proportion of episodes during which a procedure was performed ranged from $4 \%-7 \%$ in the pre-COVID-19 period and $1 \%-5 \%$ in the post-COVID-19 period. 
Table 1 displays the demographic data of patients who had a video visit in the pre-COVID-19 period $(n=2284)$ and patients who had a video visit in the post-COVID-19 period $(n=12,946)$. In the post-COVID-19 period, more black/African American (531 [4.1\%] vs 78 [3.4\%]; $P<.001)$, Hispanic/Latino (1450 [11.2\%] vs 215 [9.4\%]; $P<.001)$, and Asian (1903 [14.7\%] vs $197[8.6 \%] ; P<.001)$ patients received care via video visits compared to the pre-COVID-19 period. There was increased
post-COVID-19 utilization of video visits for patients in urban areas $(12,014$ [92.8\%] vs 2026 [88.7\%]; $P<.001)$. We did not find any difference in the insurance status of patients using video visits during either period. In the post-COVID-19 period, first clinic encounter $(2822$ [21.8\%] vs 417 [18.3\%]; $P<.001)$ and physician-provided visits (10,590 [81.8\%] vs 1538 [67.3\%]; $P<.001)$ increased.

Figure 2. Number of clinic encounters stratified by visit type (in-person visits, procedural visits, video visits, and phone visits) from January 1 to May 31, 2020, with March 16, 2020, denoting the institution-wide transition to video visits in response to coronavirus disease (COVID-19).

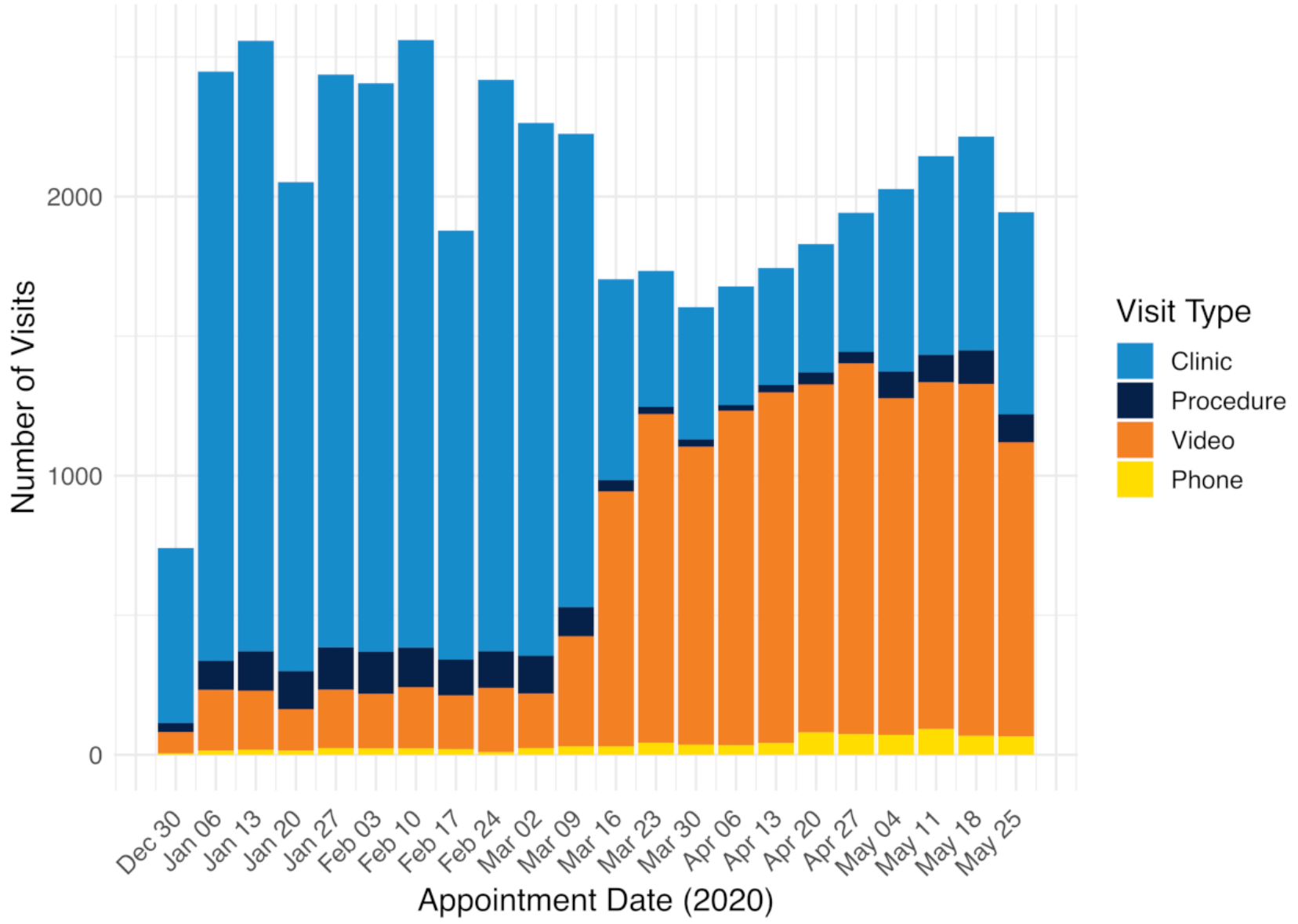


Figure 3. Relative weekly trends in clinic encounters, stratified by visit type (in-person visits, procedural visits, video visits, and phone visits) from January 1 to May 31, 2020, with March 16, 2020, denoting the institution-wide transition to video visits in response to coronavirus disease (COVID-19).

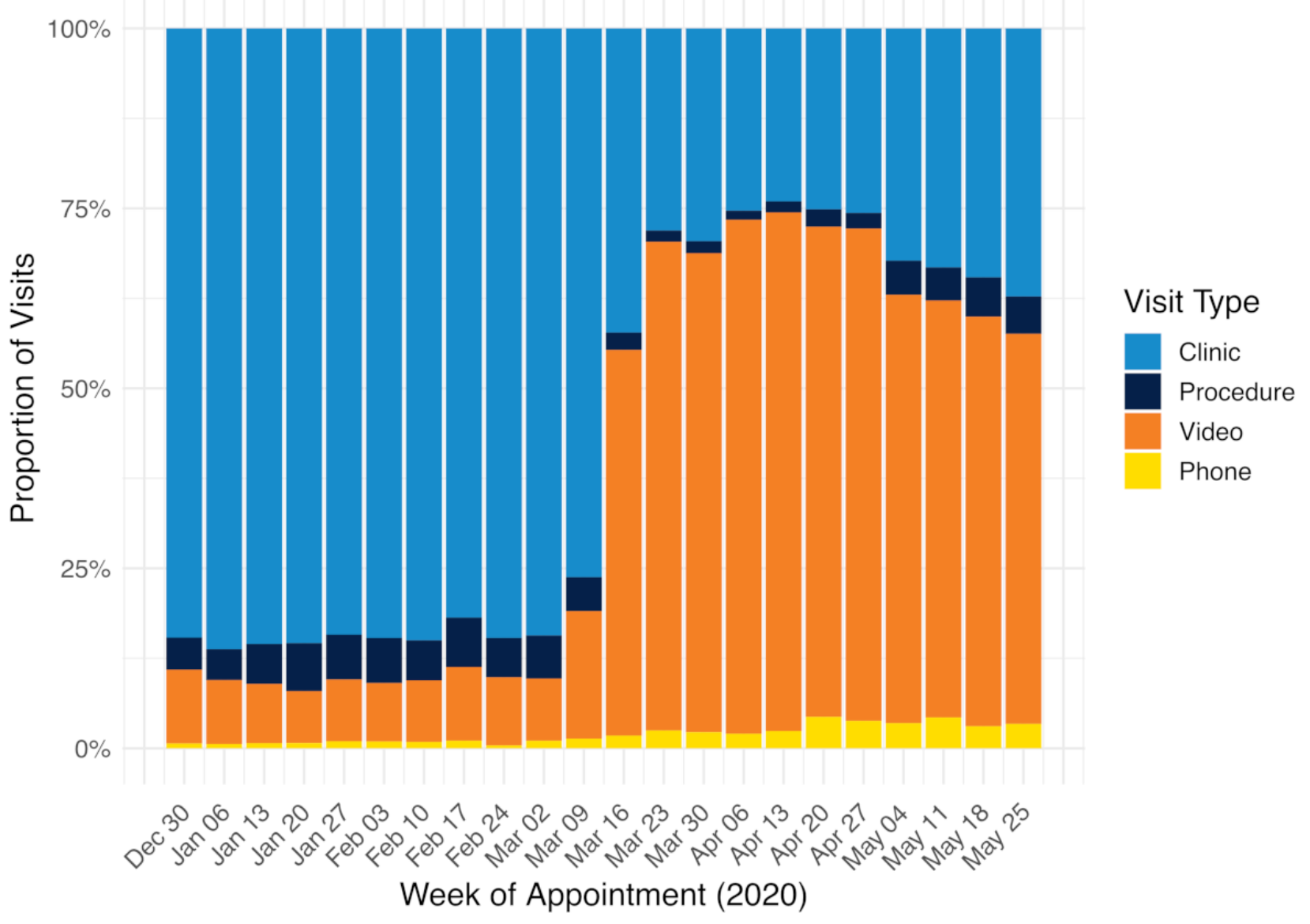


Table 1. Demographics of patients who had video visits pre- and post-COVID-19.

\begin{tabular}{|c|c|c|c|}
\hline Characteristic & Pre-COVID-19 period $(n=2284)$ & Post-COVID-19 period $(\mathrm{n}=12,946)$ & $P$ value \\
\hline Age (years), median (IQR) & $64.3(54.9-71.5)$ & $63.6(52.8-71.8)$ & .11 \\
\hline Female, n (\%) & $844(37)$ & $6123(47.3)$ & $<.001$ \\
\hline Ethnicity/race, n (\%) & & & $<.001$ \\
\hline White & $1606(70.3)$ & $7988(61.7)$ & \\
\hline Black/African American & $78(3.4)$ & $531(4.1)$ & \\
\hline Hispanic/Latino & $215(9.4)$ & $1450(11.2)$ & \\
\hline Asian & $197(8.6)$ & $1903(14.7)$ & \\
\hline Other/unknown & $188(8.2)$ & $1075(8.3)$ & \\
\hline Primary language: English & $2212(96.9)$ & $11,962(92.4)$ & $<.001$ \\
\hline Interpreter requested & $84(3.7)$ & $945(7.3)$ & $<.001$ \\
\hline Marital status, n (\%) & & & .34 \\
\hline Married/partnered & $1580(69.2)$ & $8415(65.0)$ & \\
\hline Single/separated & $373(16.3)$ & $2369(18.3)$ & \\
\hline Unknown/declined & $108(4.7)$ & $583(4.5)$ & \\
\hline Patient residence, n (\%) & & & $<.001$ \\
\hline Urban & $2026(88.7)$ & $12,014(92.8)$ & \\
\hline Rural & $255(11.2)$ & $919(7.1)$ & \\
\hline Payor, n (\%) & & & .14 \\
\hline Commercial & $929(40.7)$ & $5398(41.7)$ & \\
\hline Medicare & $1077(47.2)$ & $5709(44.1)$ & \\
\hline Medicaid & $224(9.8)$ & $1541(11.9)$ & \\
\hline Self-pay & $32(1.4)$ & $155(1.2)$ & \\
\hline First clinic encounter & $417(18.3)$ & $2822(21.8)$ & $<.001$ \\
\hline First video visit & $1054(46.2)$ & $5865(45.3)$ & .51 \\
\hline Provider type, n (\%) & & & $<.001$ \\
\hline Physician & $1538(67.3)$ & $10,590(81.8)$ & \\
\hline Advanced practice provider & $746(32.7)$ & $2356(18.2)$ & \\
\hline
\end{tabular}

\section{Discussion}

\section{Principal Findings}

We demonstrate a rapid expansion (from $<20 \%$ to $72 \%$ ) in telehealth use in a comprehensive cancer center over a remarkably brief time period in response to COVID-19 without differences in race or insurance type. Medicare telehealth visits have increased by more than $25 \%$ annually for the past decade [7], yet absolute adoption numbers remain low and fragmented with concerns about potentiating disparities in health care access [8]. The vast majority of cancer care cannot be delayed and the COVID-19 pandemic has presented new challenges that telehealth is uniquely situated to solve. Changes that would typically encompass months of planning, pilot testing, and education have been compressed into days. The use of telehealth has grown exponentially with some practices transitioning to near-complete virtual care in as little as a few days [9-11].
Several factors have likely enabled the rapid expansion of video visits at our institution. First, we had an established telehealth structure and workflow familiar to providers and practice staff. Second, UCSF made a strategic decision to provide work Relative Value Unit (wRVU) credit to providers for telehealth visits since 2015, irrespective of payer reimbursement. Third, new regulatory changes at the federal and state levels as a response to COVID-19 have reduced barriers, including the ability to see new patients (including Medicare beneficiaries) without a prior in-person visit to establish care and reimbursement for telehealth encounters by the Centers for Medicare \& Medicaid Services (CMS) at parity with in-person visits beginning March 17, 2020 [12]. Finally, CMS now permits providers licensed in any state to provide telehealth services across the country [13].

\section{Limitations}

There are a number of limitations to this study that need to be acknowledged. We did not evaluate the outcome of the video visits (eg, patient satisfaction and qualitative or clinical 
outcomes). The study is from a single, large, urban academic cancer center in the United States and our findings may not be generalizable to other specialties, practices, or locations. We believe that this is the first report of the utilization of video visits in a comprehensive cancer center in response to COVID-19 with a detailed description of the changing demographic of patients utilizing video visits before and after the COVID-19 pandemic.

\section{Conclusions}

Overall, the proportion of video visits increased from $7 \%-18 \%$ to $54 \%-72 \%$ between the pre- and post-COVID-19 periods while maintaining access to complex oncologic care at a time of social distancing. The COVID-19 pandemic has forced us to radically rethink and change our cancer care delivery models. In many health systems, there will undoubtedly be many lessons learned from this "natural experiment," which has the potential to permanently change care delivery patterns.

\section{Authors' Contributions}

AYO had full access to all of the data in the study and takes responsibility for the integrity of the data and the accuracy of the data analysis. AYO, PEL, and SLW were responsible for study concept and design; and acquisition, statistical analysis, and interpretation of data. PEL and SLW drafted the manuscript. Administrative, technical, and material support was provided by LB, and supervision was provided by PRC, RSP, and AYO. All authors critically reviewed the manuscript for important intellectual content.

\section{Conflicts of Interest}

AYO was a consultant for VSee from December 2019 to January 2020.

\section{References}

1. Hollander JE, Carr BG. Virtually Perfect? Telemedicine for Covid-19. N Engl J Med 2020 Apr 30;382(18):1679-1681. [doi: 10.1056/NEJMp2003539] [Medline: 32160451]

2. Greenhalgh T, Wherton J, Shaw S, Morrison C. Video consultations for covid-19. BMJ 2020 Mar 12;368:m998. [doi: 10.1136/bmj.m998] [Medline: 32165352]

3. Dorsey ER, Topol EJ. State of Telehealth. N Engl J Med 2016 Jul 14;375(2):154-161. [doi: 10.1056/NEJMra1601705] [Medline: 27410924]

4. Hazin R, Qaddoumi I. Teleoncology: current and future applications for improving cancer care globally. The Lancet Oncology 2010 Feb;11(2):204-210. [doi: 10.1016/s1470-2045(09)70288-8]

5. Pennell NA, Dicker AP, Tran C, Jim HSL, Schwartz DL, Stepanski EJ. mHealth: Mobile Technologies to Virtually Bring the Patient Into an Oncology Practice. Am Soc Clin Oncol Educ Book 2017;37:144-154 [FREE Full text] [doi: 10.1200/EDBK 176093] [Medline: 28561720]

6. R Core Team. A language and environment for statistical computing. Vienna, Austria: R Foundation for Statistical Computing URL: https://www.R-project.org/

7. Mehrotra A, Jena AB, Busch AB, Souza J, Uscher-Pines L, Landon BE. Utilization of Telemedicine Among Rural Medicare Beneficiaries. JAMA 2016 May 10;315(18):2015-2016. [doi: 10.1001/jama.2016.2186] [Medline: 27163991]

8. Wilcock AD, Rose S, Busch AB, Huskamp HA, Uscher-Pines L, Landon B, et al. Association Between Broadband Internet Availability and Telemedicine Use. JAMA Intern Med 2019 Jul 29;179(11):1580. [doi: 10.1001/jamainternmed.2019.2234] [Medline: $\underline{\text { 31355849] }}$

9. Mehrotra A, Ray K, Brockmeyer D, Barnett M, Bender J. Rapidly Converting to "Virtual Practices": Outpatient Care in the Era of Covid-19. 2020. URL: https://catalyst.nejm.org/doi/full/10.1056/CAT.20.0091 [accessed 2020-06-14]

10. Spiegelman J, Krenitsky N, Syeda S, Sutton D, Moroz L. Rapid Development and Implementation of a Covid-19 Telehealth Clinic for Obstetric Patients. 2020. URL: https://catalyst.nejm.org/doi/full/10.1056/CAT.20.0170 [accessed 2020-06-14]

11. Kasle DA, Torabi SJ, Savoca EL, Judson BL, Manes RP. Outpatient Otolaryngology in the Era of COVID-19: A Data-Driven Analysis of Practice Patterns. Otolaryngol Head Neck Surg 2020 May 12:194599820928987 [FREE Full text] [doi: 10.1177/0194599820928987] [Medline: 32393101]

12. Medicare Telemedicine Health Care Provider Fact Sheet. URL: https:/www.cms.gov/newsroom/fact-sheets/ medicare-telemedicine-health-care-provider-fact-sheet [accessed 2020-06-14]

13. COVID-19 Emergency Declaration Health Care Providers Fact Sheet. URL: $\underline{\text { https://www.cms.gov/files/document/ }}$ covid19-emergency-declaration-health-care-providers-fact-sheet.pdf [accessed 2020-06-14]

\section{Abbreviations}

CMS: Centers for Medicare \& Medicaid Services

COVID-19: coronavirus disease

HIPAA: Health Insurance Portability and Accountability Act

UCSF: University of California, San Francisco

wRVU: work Relative Value Unit 
Edited by G Eysenbach; submitted 15.04.20; peer-reviewed by I Mircheva, A Behmanesh, G Levin; comments to author 11.06.20; revised version received 16.06.20; accepted 21.06.20; published 06.07.20

Please cite as:

Lonergan PE, Washington III SL, Branagan L, Gleason N, Pruthi RS, Carroll PR, Odisho AY

Rapid Utilization of Telehealth in a Comprehensive Cancer Center as a Response to COVID-19: Cross-Sectional Analysis

J Med Internet Res 2020;22(7):e19322

URL: https://www.jmir.org/2020/7/e19322

doi: $\underline{10.2196 / 19322}$

PMID: $\underline{32568721}$

CPeter E Lonergan, Samuel L Washington III, Linda Branagan, Nathaniel Gleason, Raj S Pruthi, Peter R Carroll, Anobel Y Odisho. Originally published in the Journal of Medical Internet Research (http://www.jmir.org), 06.07.2020. This is an open-access article distributed under the terms of the Creative Commons Attribution License (https://creativecommons.org/licenses/by/4.0/), which permits unrestricted use, distribution, and reproduction in any medium, provided the original work, first published in the Journal of Medical Internet Research, is properly cited. The complete bibliographic information, a link to the original publication on http://www.jmir.org/, as well as this copyright and license information must be included. 